\title{
Antibiotic Resistance Trends of Nasal Staphylococcal Isolates from Namibian School Children
}

\author{
Sunette Walter, Ronnie Böck \\ Biological Sciences Department, University of Namibia, Windhoek, Namibia \\ Email: sunette.walter8@gmail.com
}

\begin{abstract}
How to cite this paper: Walter, S. and Böck, R. (2020) Antibiotic Resistance Trends of Nasal Staphylococcal Isolates from Namibian School Children. Journal of Biosciences and Medicines, 8, 18-27.

https://doi.org/10.4236/jbm.2020.83003
\end{abstract}

Received: December 18, 2019

Accepted: February 29, 2020

Published: March 3, 2020

Copyright (c) 2020 by author(s) and Scientific Research Publishing Inc. This work is licensed under the Creative Commons Attribution International License (CC BY 4.0).

http://creativecommons.org/licenses/by/4.0/

\begin{abstract}
Nasal colonization with Staphylococcus strains puts children at risk of developing difficult-to-treat staphylococcal infections. Antibiotic resistance data is limited in Namibia. Our study thus aimed to provide resistance trends for nasal staphylococci isolated from school children in the Mariental District. This is the first report on antibiotic resistance trends of staphylococci from Namibian school children. By Kirby-Bauer disk diffusion assay, 352 Staphylococcus aureus and 81 coagulase-negative staphylococci (CoNS) isolates from Namibian school children aged 6 - 14 years underwent susceptibility testing against seven antibiotics. Ninety-six percent $S$. aureus and $66.7 \%$ CoNS were resistant to ampicillin. Ampicillin resistance was significantly higher in $S$. aureus than in CoNS $(\mathrm{P}<0.0001)$. Ciprofloxacin and gentamicin were the most effective against $S$. aureus. Ciprofloxacin was also the most effective drug against CoNS. Cefoxitin/methicillin resistance was seen in $14.5 \%$ S. aureus isolates and $8.6 \%$ of CoNS. Thirty-one antibiotic resistance patterns were observed, most frequently ampicillin (A), ampicillin-erythromycin (AP-E), and ampicillin-tetracycline (AP-T). Altogether $12.5 \%$ isolates (50 S. aureus and four CoNS) were multi-drug resistant. From the methicillin-resistant $S$. aureus (MRSA) isolates, $43.1 \%$ were multi-drug resistant. Methicillin-resistant CoNS were not multi-drug resistant, with the most common resistance pattern being ampicillin-rifampicin-cefoxitin (AP-RP-FOX). In conclusion, multi-drug resistance in our study was relatively low. However, some of the MRSA isolates were multi-drug resistant, which is of concern. Learners should be educated on the importance of handwashing and appropriate use of antibiotics to prevent spread of antibiotic-resistant bacteria within the community. Ciprofloxacin and gentamicin may effectively be used to treat staphylococcal infections in this study population.
\end{abstract}




\section{Keywords}

Staphylococcus, Resistance Trends, Children, Namibia

\section{Introduction}

Healthy school children under 16 years are potential carriers of Staphylococcus aureus, especially methicillin-resistant $S$. aureus (MRSA) and multi-drug resistant strains [1]. According to [2], children are asymptomatic reservoirs for community-associated MRSA (CA-MRSA) which enables these bacteria to rapidly spread within communities. Nasal colonization with $S$. aureus is a major risk factor for staphylococcal infection [3]. Most staphylococcal infections can easily be cleared with antibiotics, but bacteria that develop resistance towards certain antibiotics make treatment options limited, especially if antibiograms for reference purposes are unavailable. Methicillin-resistant $S$. aureus is resistant to beta-lactam antibiotics, while some strains are multi-drug resistant. Drug resistant strains are often responsible for chronic, persistent and recurrent infections, which is a challenge for healthcare practitioners.

Antibiotic resistance data is limited in Namibia and few reports on staphylococcal drug resistance exist. Our study aimed to make a contribution towards closing this gap in information by providing resistance data for $433 \mathrm{~S}$. aureus and coagulase-negative staphylococci (CoNS) isolates from nasal swabs of children aged 6 - 14 years attending schools in the Mariental District. To our knowledge, this is the first report on resistance trends of nasal staphylococcal isolates from Namibian school children.

\section{Materials and Methods}

\subsection{Study Area, Population and Sample Collection}

This was a cross-sectional study in the town of Mariental, located southeast of Namibia's capital city of Windhoek on the B1 national highway. With written consent from their parents/guardians, the population that was screened for nasal staphylococci consisted of randomly chosen healthy learners attending five schools in the Mariental District. The children were divided into two age-groups: 6 - 10 years and $11-14$ years, and consisted of 126 boys and 146 girls. Sample collection was done during March, September and October 2016. One nasal specimen was obtained from each child by gently rotating a sterile Amies transport medium swab (Labocare ${ }^{\mathrm{TM}}$, Johannesburg, South Africa) thoroughly around the perimeter of both nostrils. Specimens were kept frozen at $-20^{\circ} \mathrm{C}$ until transporting them to the University of Namibia's Biomedical Research Laboratory for processing.

\subsection{Bacterial Cultures Used}

Two commercially obtained reference strains, $S$. aureus ATCC 25923 (an antibi- 
otic susceptible strain) and $S$. aureus ATCC 33591 (a multi-drug resistant MRSA strain\} (Microbiologics ${ }^{\oplus}$, St. Cloud, US), as well as 433 staphylococcal nasal isolates from 272 Mariental school children, were used in antibiotic assays. Of these isolates, 352 were $S$. aureus and 81 were CoNS.

\subsection{Antibiotic Susceptibility Testing}

Antibiotic resistance testing on isolates obtained from the nasal specimens was carried out in 2017. The Kirby-Bauer disk diffusion assay was used to determine antibiotic susceptibility/resistance in isolates [4] [5] [6] [7]. Table 1 shows the antibiotics that were used and interpretation of inhibition zones. Staphylococcus aureus ATCC 25923, S. aureus ATCC 33591 (MRSA), 352 nasal S. aureus isolates and 81 nasal CoNS were tested. Resistance towards cefoxitin $(30 \mu \mathrm{g})$, in other words, an inhibition zone diameter $<22 \mathrm{~mm}$, indicated MRSA or methicillin-resistant CoNS (MRCoNS), while resistance towards three or more different classes of antibiotics was an indication of multi-drug resistance in bacteria. By direct colony suspension, three to five well-isolated colonies from overnight tryptone soy agar (Scharlau Microbiology, Spain) plate cultures were inoculated into $10 \mathrm{ml}$ sterile phosphate-buffered saline $\mathrm{pH} 6.8$ - 7.4 (Skylabs, Johannesburg, $\mathrm{SA})$ and adjusted to $0.5 \mathrm{McF}$ arland standard $\left(1.5 \times 10^{8} \mathrm{CFU} / \mathrm{ml}\right.$, absorbance reading $0.08-0.13$ at $625 \mathrm{~nm}$ ). Adjusted cultures were then swabbed onto Mueller-Hinton agar (Mast Diagnostics, Merseyside, UK) and left to dry for 5 - 10 minutes at room temperature before dispensing the antibiotic disks (Mast Diagnostics, Merseyside, UK) onto the plates. Plates were incubated at $35^{\circ} \mathrm{C}$ for 18 - 20 hours and diameters were measured using a ruler. Isolates were classified as susceptible, resistant or intermediately resistant towards each antibiotic, according to the diameter (in millimetres) of their zones of inhibition. The reference strains $S$. aureus ATCC 25923 (susceptible) and $S$. aureus ATCC 33591

Table 1. Antibiotics used in this study and interpretation of inhibition zones of test cultures. Adapted from [6] and [7].

\begin{tabular}{|c|c|c|c|c|c|c|}
\hline Chemical class & Antibiotic & $\begin{array}{c}\text { Disk } \\
\text { symbol }\end{array}$ & $\begin{array}{c}\text { Disk } \\
\text { content }\end{array}$ & Resistant & Intermediate & Susceptible \\
\hline \multirow{4}{*}{ Aminoglycosides } & \multirow{4}{*}{ Gentamicin } & \multirow{4}{*}{ GM } & \multirow{4}{*}{$10 \mu \mathrm{g}$} & $<18 \mathrm{~mm}$ & \multirow{4}{*}{-} & $\geq 18 \mathrm{~mm}$ \\
\hline & & & & $S$. aureus & & $S$. aureus \\
\hline & & & & $<22 \mathrm{~mm}$ & & $\geq 22 \mathrm{~mm}$ \\
\hline & & & & CoNS & & CoNS \\
\hline$\beta$-lactams & Ampicillin & AP & $25 \mu \mathrm{g}$ & $<18 \mathrm{~mm}$ & - & $\geq 18 \mathrm{~mm}$ \\
\hline $\begin{array}{l}\text { Cephalosporins } \\
\text { (also a } \beta \text {-lactam) }\end{array}$ & Cefoxitin & FOX & $30 \mu \mathrm{g}$ & $<22 \mathrm{~mm}$ & - & $\geq 22 \mathrm{~mm}$ \\
\hline Fluoroquinolones & Ciprofloxacin & CIP & $5 \mu \mathrm{g}$ & $<20 \mathrm{~mm}$ & - & $\geq 20 \mathrm{~mm}$ \\
\hline Macrolides & Erythromycin & $\mathrm{E}$ & $15 \mu \mathrm{g}$ & $<18 \mathrm{~mm}$ & $18-20 \mathrm{~mm}$ & $\geq 21 \mathrm{~mm}$ \\
\hline Tetracyclines & Tetracycline & $\mathrm{T}$ & $30 \mu \mathrm{g}$ & $<19 \mathrm{~mm}$ & $19-21 \mathrm{~mm}$ & $\geq 22 \mathrm{~mm}$ \\
\hline Other & Rifampicin & $\mathrm{RP}$ & $5 \mu \mathrm{g}$ & $<23 \mathrm{~mm}$ & $23-25 \mathrm{~mm}$ & $\geq 26 \mathrm{~mm}$ \\
\hline
\end{tabular}


(multi-drug resistant MRSA) served as quality control strains. Clinical and Laboratory Standards Institute (CLSI) guidelines [6] and the European Committee on Antimicrobial Susceptibility Testing (EUCAST) breakpoint tables [7] were used to interpret results.

\subsection{Statistical Analysis}

A chi-square test for comparison of proportions with MedCalc statistical software (MedCalc statistical software version 16.4.3 \{MedCalc software bvba, Ostend, Belgium; https://www.medcalc.org; 2016\}) [8] was used to compare percentage differences in antibiotic resistance between $S$. aureus and CoNS. Statistical significant differences were indicated by a P-value of $\leq 0.05$.

\section{Results and Discussion}

\subsection{Antibiotic Susceptibility/Resistance of Isolates}

Altogether 433 staphylococcal isolates, as well as two reference strains, underwent antibiotic susceptibility testing against seven antibiotics (Table 1). Excluding the reference strains, 352 of these were $S$. aureus and 81 were CoNS. Only $4.8 \%$ of isolates were susceptible to all antibiotics tested. As expected, $S$. aureus ATCC 25923 was susceptible to all antibiotics, while $S$. aureus ATCC 33591 (MRSA) was resistant to cefoxitin and also multi-drug resistant.

Table 2 summarizes percentage susceptibility, intermediacy or resistance of the 352 S. aureus isolates to the antibiotics. Most isolates (96.0\%) were resistant to ampicillin, rendering this antibiotic mostly ineffective against $S$. aureus. For CoNS, $66.7 \%$ of isolates were also resistant to ampicillin (Table 3 ). However, resistance towards ampicillin was significantly higher in $S$. aureus than in CoNS (P $<0.0001)$. Resistance towards ampicillin has become very common in staphylococci and can be ascribed to the action of the enzyme beta-lactamase which is under plasmid control. Plasmids containing resistance genes can be transferred from one bacterium to another [9]. Other studies also indicated high ampicillin resistance in $S$. aureus, in northeastern Brazil, for example, de Carvalho et al., 2017 [10] observed $80.0 \%$ ampicillin resistance in $S$. aureus from nasal secretions of children attending public daycare. In our study, ampicillin was still effective against $33.3 \%$ of coagulase-negative isolates. Ampicillin should not be the drug of choice for treating infections caused by $S$. aureus and CoNS, unless used in combination with other drugs.

As indicated in Table 2 and Table 3, respectively, a total of 83 (23.6\%) S. aureus isolates and nine (11.1\%) CoNS were resistant to erythromycin $(\mathrm{P}=$ 0.0064). These resistance rates are not that high and erythromycin is therefore expected to be effective against staphylococci in most instances. In comparison to our study, a study by Mengistu et al., 2013 [11] using Namibia Institute of Pathology (NIP) data showed higher resistance (32.3\%) to erythromycin in Staphylococcus isolated from cerebrospinal fluid (CSF) for the period 2009-2012. Another Namibian study undertaken by Iileka et al., 2016 [12] reported a lower 
Table 2. Percentage susceptibility, intermediacy or resistance for $S$. aureus isolates (n = $352)$.

\begin{tabular}{cccc}
\hline Antibiotic & Susceptible (\%) & Intermediate (\%) & Resistant (\%) \\
\hline Ampicillin $10 \mu \mathrm{g}$ & $14(4.0)$ & - & $338(96.0)$ \\
Cefoxitin $30 \mu \mathrm{g}$ & $301(85.5)$ & - & $51(14.5)$ \\
Ciprofloxacin $5 \mu \mathrm{g}$ & $351(99.7)$ & - & $1(0.3)$ \\
Erythromycin $15 \mu \mathrm{g}$ & $238(67.6)$ & $31(8.8)$ & $83(23.6)$ \\
Gentamicin $10 \mu \mathrm{g}$ & $328(93.2)$ & - & $24(6.8)$ \\
Rifampicin $5 \mu \mathrm{g}$ & $248(70.4)$ & $40(11.4)$ & $64(18.2)$ \\
Tetracycline $30 \mu \mathrm{g}$ & $232(66.0)$ & $60(17.0)$ & $60(17.0)$ \\
\hline
\end{tabular}

Table 3. Percentage susceptibility, intermediacy or resistance for CoNS isolates $(n=81)$.

\begin{tabular}{cccc}
\hline Antibiotic & Susceptible (\%) & Intermediate (\%) & Resistant (\%) \\
\hline Ampicillin $10 \mu \mathrm{g}$ & $27(33.3)$ & - & $54(66.7)$ \\
Cefoxitin $30 \mu \mathrm{g}$ & $74(91.4)$ & - & $7(8.6)$ \\
Ciprofloxacin $5 \mu \mathrm{g}$ & $81(100.0)$ & - & $0(0)$ \\
Erythromycin $15 \mu \mathrm{g}$ & $65(80.2)$ & $8(9.9)$ & $8(9.9)$ \\
Gentamicin $10 \mu \mathrm{g}$ & $72(88.9)$ & - & $9(11.1)$ \\
Rifampicin $5 \mu \mathrm{g}$ & $55(67.9)$ & $12(14.8)$ & $14(17.3)$ \\
Tetracycline $30 \mu \mathrm{g}$ & $60(74.1)$ & $14(17.3)$ & $7(8.6)$ \\
\hline
\end{tabular}

erythromycin percentage resistance (10.2\%) than ours for $S$. aureus isolates obtained from various clinical samples over the time period 2012-2014. According to PathCare Namibia data [13] $11.0 \% S$. aureus bacteria were resistant to erythromycin from 2014 to 2015 , which is very close to the $10.2 \%$ indicated by Iileka et al., 2016 [12]. In Brazil de Carvalho et al., 2017 [10] observed a higher percentage (32.8\%) erythromycin resistance for $S$. aureus compared to our results. Their percentage resistance is however almost the same as the $32.3 \%$ from Mengistu et al., 2013 [11].

In the current study, only $17.0 \%$ S. aureus and $8.6 \%$ CoNS $(\mathrm{P}=0.0595)$ were resistant to tetracycline, indicating its effectiveness against Staphylococcus. According to Mengistu et al., 2013 [11] 29.6\% of staphylococci from CSF displayed resistance towards tetracycline. In agreement with our results, Iileka et al., 2016 [12] reported $17.4 \%$ tetracycline resistance in clinical $S$. aureus strains across the period 2012-2014. The resistance for $S$. aureus in our study towards this antibiotic is higher than the 4.3\% found by de Carvalho et al., 2017 [10] in Brazil.

Rifampicin resistance in our study was relatively low at $18.2 \%$ and $17.3 \%(\mathrm{P}=$ 0.8495) for $S$. aureus and CoNS isolates, respectively. However, this is somewhat higher than the 7.0\% indicated in other Namibian resistance data [13]. Rifampicin resistance in other countries may be higher. In India for instance, Bharathi et al., 2014 [14] observed 64.7\% resistance in nasal MRSA from school children. 
Rifampicin is among the antibiotics commonly used to treat MRSA infections in India [14]. Staphylococci can quickly develop resistance to rifampicin [9].

According to our results, ciprofloxacin and gentamicin were most effective against $S$. aureus, with $99.7 \%$ and $93.2 \%$ of isolates that were susceptible to these drugs, respectively. Ciprofloxacin was also the most effective drug against CoNS, with $100.0 \%$ susceptibility. Resistance between $S$. aureus and CoNS for the two antibiotics was not statistically significant $(\mathrm{P}=0.6220 ; \mathrm{P}=0.1886)$. In line with our results, de Carvalho et al., 2017 [10] observed 92.9\% S. aureus susceptibility towards ciprofloxacin in Brazil. In previous Namibian studies Mengistu et al., 2013 [11] reported 19.0\% ciprofloxacin resistance and 52.9\% gentamicin resistance for Staphylococcus from CSF samples, whereas Iileka et al., 2016 [12] reported a low $4.4 \%$ ciprofloxacin resistance for $S$. aureus clinical isolates. Namibian susceptibility data for 2011-2012 [13] showed $S$. aureus to be $96.0 \%$ susceptible to ciprofloxacin, while data for 2014-2015 indicated $95.0 \%$ susceptibility to gentamicin. Based on our findings, ciprofloxacin and gentamicin could be used to treat certain staphylococcal infections in our study population. However, taking into consideration the $52.9 \%$ resistance towards gentamicin in CSF Staphylococcus observed by lileka et al., 2016 [12], care should be taken by medical doctors when prescribing gentamicin for meningitis in Namibia.

In this study, cefoxitin was used to detect methicillin-resistant bacteria. These bacteria are also resistant to all beta-lactam antibiotics. A total of 51/352 (14.5\%) $S$. aureus isolates were resistant to cefoxitin and therefore identified as MRSA, whereas 7/81 (8.6\%) of CoNS isolates were MRCoNS. Cefoxitin resistance between $S$. aureus and CoNS were statistically insignificant $(\mathrm{P}=0.1603)$. Our MRSA prevalence is close to the $13.8 \%$ cefoxitin-resistant $S$. aureus reported by Reta et al., 2015 [15] in a community based cross-sectional study that involved children aged 6 - 12 years from nine primary schools in Bahir Dar Town, Ethiopia. It is also in line with other Namibian data that showed $13.6 \%$ MRSA (2010-2014) and 13.5\% MRSA (2012-2014) obtained from various clinical specimens by Festus et al., 2016 [16] and Iileka et al., 2016 [12]. Considering these relatively low percentages, MRSA does not seem to be a major problem in $\mathrm{Na}$ mibia yet. In general, there are not many studies available on methicillin resistance in CoNS. According to [17], MRSA and MRCoNS can be found together in the human nose and have similar antibiotics resistance genes that can be transferred between bacteria. We could not find studies involving nasal antibiotic resistant staphylococci from healthy school children in our neighboring countries (Angola, Zambia, Botswana and South Africa) to compare our results with.

\subsection{Resistance Patterns and Multi-Drug Resistance}

Altogether 31 antibiotic resistance patterns were observed in this study (Table 4). Isolates with similar resistance patterns could be considered the same strain, unless they acquired these resistance genes from other strains, therefore sharing the same resistance pattern. For $S$. aureus, 27 different resistance patterns were obtained. For CoNS, there were 14 different patterns. Ten patterns (AP, RP, 
Table 4. Resistance patterns for $433 S$. aureus and CoNS isolates from school children aged 6 - 14 years, against seven antibiotics.

\begin{tabular}{|c|c|c|c|}
\hline $\begin{array}{l}\text { Number of isolates } \\
\text { with this pattern }\end{array}$ & $\begin{array}{l}\text { Number of } S \text {. aureus } \\
\text { isolates with this pattern }\end{array}$ & $\begin{array}{l}\text { Number of CoNS isolates } \\
\text { with this pattern }\end{array}$ & $\begin{array}{c}\text { Antibiotic } \\
\text { resistance pattern }\end{array}$ \\
\hline 194 & 162 & 32 & $\mathrm{AP}$ \\
\hline 2 & 0 & 2 & $\mathrm{E}$ \\
\hline 2 & 0 & 2 & GM \\
\hline 4 & 3 & 1 & $\mathrm{RP}$ \\
\hline 2 & 0 & 2 & $\mathrm{~T}$ \\
\hline 52 & 50 & 2 & AP-E \\
\hline 19 & 14 & 5 & AP-GM \\
\hline 11 & 8 & 3 & AP-RP \\
\hline 23 & 22 & 1 & AP-T \\
\hline 8 & 8 & 0 & AP-FOX \\
\hline 1 & 1 & 0 & GM-E \\
\hline 2 & 2 & 0 & T-RP \\
\hline 1 & 1 & 0 & RP-E \\
\hline 1 & 1 & 0 & AP-CIP-FOX \\
\hline 5 & 5 & 0 & AP-E-FOX \\
\hline 3 & 3 & 0 & AP-GM-E \\
\hline 5 & 4 & 1 & AP-GM-RP \\
\hline 1 & 1 & 0 & AP-GM-T \\
\hline 4 & 4 & 0 & AP-RP-E \\
\hline 19 & 12 & 7 & AP-RP-FOX \\
\hline 9 & 8 & 1 & AP-T-E \\
\hline 7 & 7 & 0 & AP-T-RP \\
\hline 3 & 3 & 0 & AP-T-FOX \\
\hline 1 & 0 & 1 & GM-T-E \\
\hline 1 & 1 & 0 & AP-GM-RP-FOX \\
\hline 5 & 5 & 0 & AP-RP-E-FOX \\
\hline 1 & 1 & 0 & AP-T-E-FOX \\
\hline 2 & 1 & 1 & AP-T-RP-E \\
\hline 11 & 11 & 0 & AP-T-RP-FOX \\
\hline 3 & 3 & 0 & AP-T-RP-E-FOX \\
\hline 1 & 1 & 0 & AP-GM-T-RP-E-FOX \\
\hline
\end{tabular}

Key: Multi-drug resistant $\square$; Multi-drug resistant MRSA $\square$; AP-ampicillin (10 $\mu \mathrm{g})$; CIP-ciprofloxacin $(5 \mu \mathrm{g}) ;(\mathrm{E}$-erythromycin $(15 \mu \mathrm{g})$; FOX-cefoxitin $(30 \mu \mathrm{g}) ; \mathrm{GM}$-gentamicin $(10$ $\mu \mathrm{g}) ; \mathrm{RP}$-rifampicin $(5 \mu \mathrm{g})$; T-tetracycline $(30 \mu \mathrm{g})$.

AP-E, AP-GM, AP-RP, AP-T, AP-GM-RP, AP-RP-FOX, AP-T-E and AP-T-RP-E) were shared by $S$. aureus and CoNS. AP-RP-FOX was the only pattern shared 
between MRSA and MRCoNS. The three most frequently encountered patterns were AP, AP-E and AP-T, with 194, 52 and 23 isolates having these, respectively.

Fifty-four out of 433 isolates (12.5\%) were resistant against three or more classes of antibiotics and classified as multi-drug resistant. Of these, 50 isolates were $S$. aureus and four were CoNS. The most common multi-drug resistant pattern for methicillin-susceptible isolates was AP-T-E, displayed by eight $S$. aureus isolates, and one coagulase-negative isolate. Of the 51 MRSA isolates, 22 (43.1\%) were multi-drug resistant, with AP-T-RP-FOX as the most encountered resistance pattern among them (See Figure 1). This is of concern, but is 14.5\% less than the $57.6 \%$ multi-drug resistant MRSA isolated from Iranian children by Erami et al., 2014 [18], and 20.5\% less than the 63.6\% from Brazilian children as reported by de Carvalho et al., 2017 [10]. One of the multi-drug resistant MRSA isolates in our study showed resistance towards 6/7 antibiotics tested with only ciprofloxacin that was effective against it. Methicillin-resistant CoNS did not show multi-drug resistance, with the most common resistance pattern being AP-RP-FOX. Overall, our findings support the conclusion by Arali et al., 2016 [1] that healthy school children under the age of 16 years are potential carriers of MRSA and multi-drug resistant strains.

\section{Conclusion}

Our study showed the presence of antibiotic-resistant strains among healthy school children. Overall, multi-drug resistance was relatively low. However, some of the MRSA isolates were multi-drug resistant, which is of concern. Learners should be encouraged to frequently wash their hands to prevent spread of antibiotic-resistant bacteria within the Mariental community and educated on

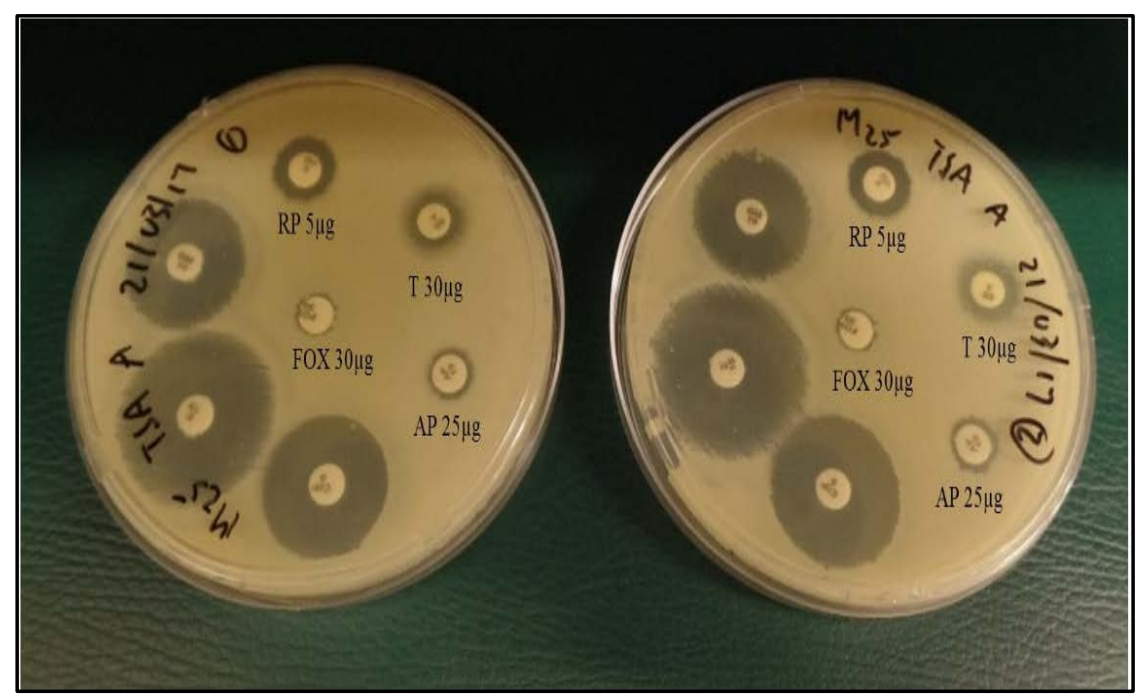

Figure 1. Duplicate Kirby-Bauer disk diffusion assay on Mueller-Hinton agar showing reduced inhibition zones of multi-drug resistant MRSA isolated from a 9-year-old girl (resistance pattern AP-T-RP-FOX). The left three germicidal circles indicate susceptibility of the bacteria against $10 \mu \mathrm{g}$ gentamicin (top), $15 \mu \mathrm{g}$ erythromycin (middle) and $5 \mu \mathrm{g}$ ciprofloxacin (bottom). 
the appropriate use of antibiotics. Ciprofloxacin and gentamicin may effectively be used to treat staphylococcal infections in this study population.

\section{Acknowledgements}

The authors acknowledge and thank the University of Namibia's Biomedical Research Laboratory, Biological Sciences Department, where the research was completed. We also acknowledge and thank the Southern African Biochemistry and Informatics for Natural Products (SABINA) and the Regional Initiative in Science and Education (RISE) for financial support. This work forms part of a larger Ph.D. study undertaken at the University of Namibia.

\section{Conflicts of Interest}

The authors declare no conflicts of interest regarding the publication of this paper.

\section{References}

[1] Arali, S.M., Kulkarni, V., Manjula, N.G., Gaddad, S.M., Jayaraj, Y.M. and Shivannavar, C.T. (2016) Prevalence and Antibiotic Susceptibility Profile of MRSA Isolated from the Anterior Nares of School Going Children in Gulbarga. International Journal of Medical Science and Public Health, 5, 620-626. https://doi.org/10.5455/ijmsph.2016.0805201592

[2] Alzoubi, H.M., Agel, A.A., Al-Sarayreh, S.A. and Al-Zayadneh, E. (2014) Methicillin-Resistant Staphylococcus aureus Nasal Carriage among Primary School-Aged Children from Jordan: Prevalence, Antibiotic Resistance and Molecular Characteristics. Journal of Egyptian Public Health Association, 9, 114-118. https://doi.org/10.1097/01.EPX.0000454671.83406.e8

[3] Reid, M.J.A., Fischer, R.S.B., Mannathoko, N., Muthoga, C., McHugh, E., Essigmann, H., Brown, E.L. and Steenhoff, A.P. (2017) Prevalence of Staphylococcus aureus Nasal Carriage in Human Immunodeficiency Virus-Infected and Uninfected Children in Botswana: Prevalence and Risk Factors. The American Journal of Tropical Medicine and Hygiene, 96, 795-801. https://doi.org/10.4269/ajtmh.16-0650

[4] Harley, J.P. and Prescott, L.M. (2002) Laboratory Exercises in Microbiology. 5th Edition, McGraw-Hill, New York.

[5] British Society for Antimicrobial Chemotherapy (BSAC) Methods for Antimicrobial Susceptibility Testing Version 6.0.

[6] Clinical and Laboratory Standards Institute (CLSI) (2013) Performance Standards for Antimicrobial Susceptibility Testing: Twenty-Third Informational Supplement. CLSI Document M100-S23. CLSI, Wayne.

[7] European Committee on Antimicrobial Susceptibility Testing (EUCAST) (2017) Breakpoint Tables for Interpretation of MICs and Zone Diameters Version 7.1. http://eucast.org

[8] MedCalc (2016) MedCalc Statistical Software Version 16.4.3. https://www.medcalc.org

[9] Brooks, G.F., Carroll, K.C., Butel, J.S., Morse, S.A. and Mietzner, T.A. (2013) Jawetz, Melnick \& Adelberg's Medical Microbiology. 26th Edition, McGraw-Hill, New York.

[10] de Carvalho, S.P., de Almeida, J.B., Andrade, Y.M.F.S., da Silva, L.S.C., de Oliveira, 
M.V., Timenetsky, J. and Marques, L.M. (2017) Community-Acquired Methicillin-Resistant Staphylococcus aureus Carrying SCCmec Type IV and V Isolated from Healthy Children Attending Public Daycare in Northeastern Brazil. The Brazilian Journal of Infectious Diseases, 21, 464-467. https://doi.org/10.1016/j.bjid.2017.04.001

[11] Mengistu, A., Gaeseb, J., Uaaka, G., Ndjavera, C., Kambyambya, K., Indongo, L., Kalemeera, F., Ntege, C., Mabirizi, D., Joshi, M.P. and Sagwa, E. (2013) Antimicrobial Sensitivity Patterns of Cerebrospinal Fluid (CSF) Isolates in Namibia: Implications for Empirical Antibiotic Treatment of Meningitis. Journal of Pharmaceutical Policy and Practice, 6, Article No. 4. https://doi.org/10.1186/2052-3211-6-4

[12] Iileka, A.E.K., Mukesi, M., Engelbrecht, F. and Moyo, S.R. (2016) Antimicrobial Susceptibility Patterns of Staphylococcus aureus Strains Isolated at the Namibia Institute of Pathology 2012 to 2014. Open Journal of Medical Microbiology, 6, 116-124. https://doi.org/10.4236/ojmm.2016.63016

[13] PathCare Namibia (2015) Antimicrobial Susceptibility Data. http://196.25.36.50/webfiles/files/ANTIMICROBIAL_SUSCEPTIBILITY_DATA_St aphylococcus_aureus.pdf

[14] Bharathi, M., Lakshmi, N., Kalyani, C.S. and Padmaja, I.J. (2014) Nasal Carriage of Multidrug-Resistant MSSA and MRSA in Children of Municipality Schools [Letter to the Editor]. Indian Journal of Medical Microbiology, 32, 200.

http://www.ijmm.org/text.asp?2014/32/2/200/129840 https://doi.org/10.4103/0255-0857.129840

[15] Reta, A., Gedefaw, L., Sewunet, T. and Beyene, G. (2015) Nasal Carriage, Risk Factors and Antimicrobial Susceptibility Pattern of Methicillin-Resistant Staphylococcus aureus among School Children in Ethiopia. Journal of Medical Microbiology and Diagnosis, 4, 177.

https://www.omicsonline.org/peer-reviewed/nasal-carriage-risk-factors-and-antimicrobi al-susceptibility-pattern-of-methicillin-resistant-staphylococcus-aureus-among-sch ool-c-42812.html

[16] Festus, T., Mukesil, M. and Moyo, S.R. (2016). The Distribution of Methicillin Resistant Staphylococcus aureus Isolated at the Namibia Institute of Pathology in Windhoek, Namibia. Indian Journal of Medical Research and Pharmaceutical Sciences, 3, 1-8.

https://pdfs.semanticscholar.org/7b04/5d14227962c4ae3671ca82eafe43ec9e062c.pdf

[17] Jayadev-Menon, P., Humphreys, H. and Fitzgerald-Hughes, D. (2015) Could Coagulase Negative Staphylococci Be an Evolutionary Source of Resistance Genes for Staphylococcus aureus? BMC Proceedings, 9, A43.

http://www.biomedcentral.com/1753-6561/9/S1/A43

https://doi.org/10.1186/1753-6561-9-S1-A43

[18] Erami, M., Soltani, B., Ardakani, A.T., Moravveji, A., Rezaei, M.H., Soltani, S. and Moniri, R. (2014) Nasal Carriage and Resistance Pattern of Multidrug Resistant Staphylococcus aureus among Healthy Children in Kashan, Iran. Iranian Red Crescent Medical Journal, 16, e21346.

https://www.researchgate.net/publication/280977442_Nasal_Carriage_Rates_of_Staphyloco ccus_aureus_and_CA-Methicillin_Resistant_Staphylococcus_aureus_among_Unive rsity_Students

https://doi.org/10.5812/ircmj.21346 